\title{
Automatic Diffraction Analysis and Lattice Fitting for Convergent-Beam Electron Diffraction Patterns in 4D-STEM
}

\author{
Sihan Wang ${ }^{1}$, Tim Eldred ${ }^{2}$, Jacob Smith $^{2}$ and Wenpei Gao ${ }^{2}$ \\ ${ }^{1}$ North Carolina State University, North Carolina, United States, ${ }^{2}$ North Carolina State University, United States
}

Recent development of four dimensional scanning transmission electron microscopy (4D-STEM) enables the capture of scanning diffraction data over a relatively large area, making analysis of local symmetry, orientation, and strain possible [1]. For detailed analysis of the diffraction patterns, accurately detecting and locating diffraction disks is important, however, challenging. Here, we show a workflow of methods to automatically locate disks in diffraction patterns, refine their positions and optimize their registries in a reciprocal lattice. Local lattice parameters and distortion can then be measured based on the reciprocal lattice. As this approach requires no prior knowledge about the material or structure, it can automatically analyze the disk registration of each diffraction pattern in a 4D data set.

Our method includes two processes, disk center detection and reciprocal lattice fitting as illustrated in Figure 1(ac) with simulated convergent beam electron diffraction (CBED) patterns using MULTEM [2]. Each pattern is first rotated to make a row of diffraction disks along the horizontal direction (Figure 1(a)), so will be the corresponding base vector of the reciprocal lattice. In disk detection, cross-correlation is applied to the CBED patterns with a ring filter, ideally, the center of each diffraction disk will show the highest cross-correlation coefficient. Then, Laplacian of Gaussian (LoG) filters with different sizes are used to detect center blobs in the cross-correlation map and export a rough center position for each disk. The detected center positions are refined using the radial gradient maximization method [3], which loops through a window around the roughly detected centers. We finally fit the refined disk positions with a reciprocal lattice. The disk centers with similar row coordinates are assigned to the same row group with averaged spacing between all neighboring disk centers calculated as the length of the first base vector. Another first-order disk closest to the zero-order disk in a different row group is then selected and its position is used to roughly estimate the second base vector. With these two vectors, a two-dimensional matrix of hypothetical lattice points is generated (Figure 1(b)) and optimized by comparing the detected disk positions with the hypothetical lattice points (Figure 1(c)). In Figure1(d-e), our method can be feasibly applied to simulated CBED patterns with high noise levels. The uncertainty of this method is analyzed by comparing the detected lattice parameters to the input structure of the simulation.

We further showcase our method for strain mapping by analyzing a big dataset of 4D-STEM acquired from a Pd@Pt core-shell octahedral nanoparticle (Figure 2. a-d). Our method identifies almost all the detectable diffraction disks which are hardly visible (Figure 2(d)). In the case where there are overlapped patterns (Figure 2(c)), our method outputs the positions of the disks fitted better in a lattice. The strain mapping of the Pd@Pt sample calculated using the method in ref [4] is shown in Figure 2(e-g). With the robustness in diffraction pattern recognition against high noise in the image and automated reciprocal lattice analysis, our method finds a wide variety of applications on structural analysis. We will discuss the details of data analysis, its limitation and improvement, and results from other materials in the presentation.[5] 

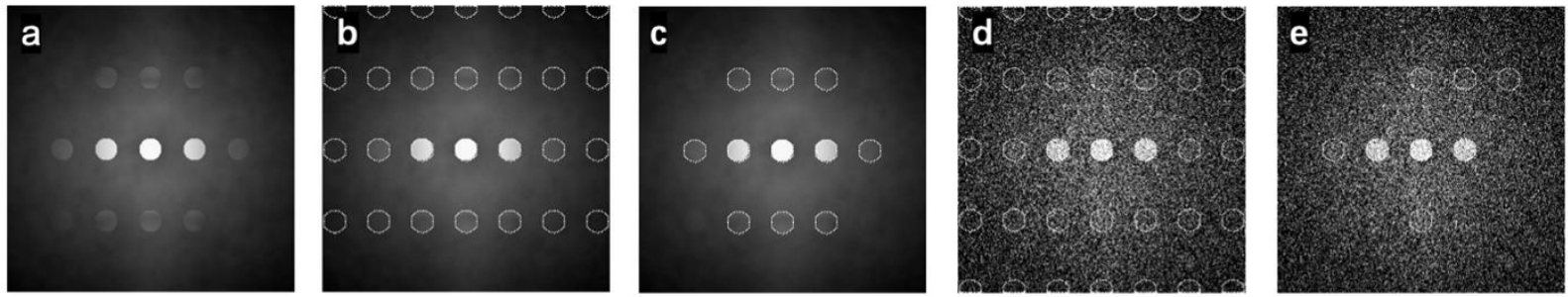

Figure 1. Illustration of the diffraction pattern analysis. (a) The simulated diffraction pattern of Pd along its [100] zone axis. (b) The fitted reciprocal lattice of (a). (c) All detected disks in the fitted reciprocal lattice. (d) The fitting of reciprocal lattice using simulated diffraction pattern with high noise. (e) All detected disks in the fitted reciprocal lattice in (d).
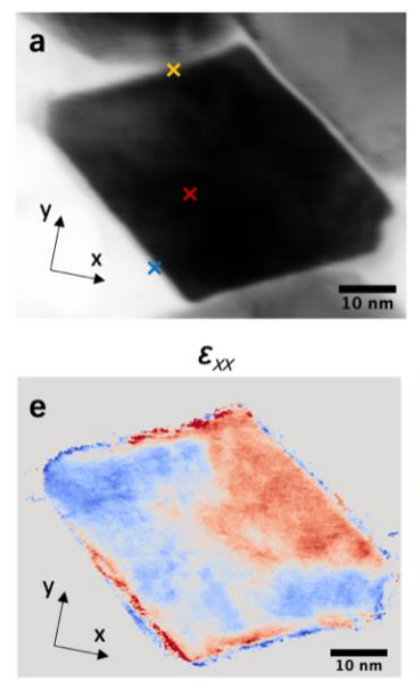

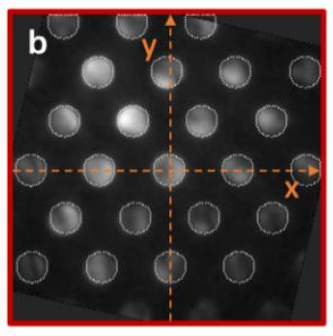

$\varepsilon_{y y}$

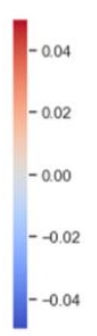

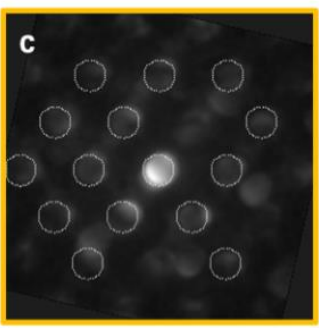
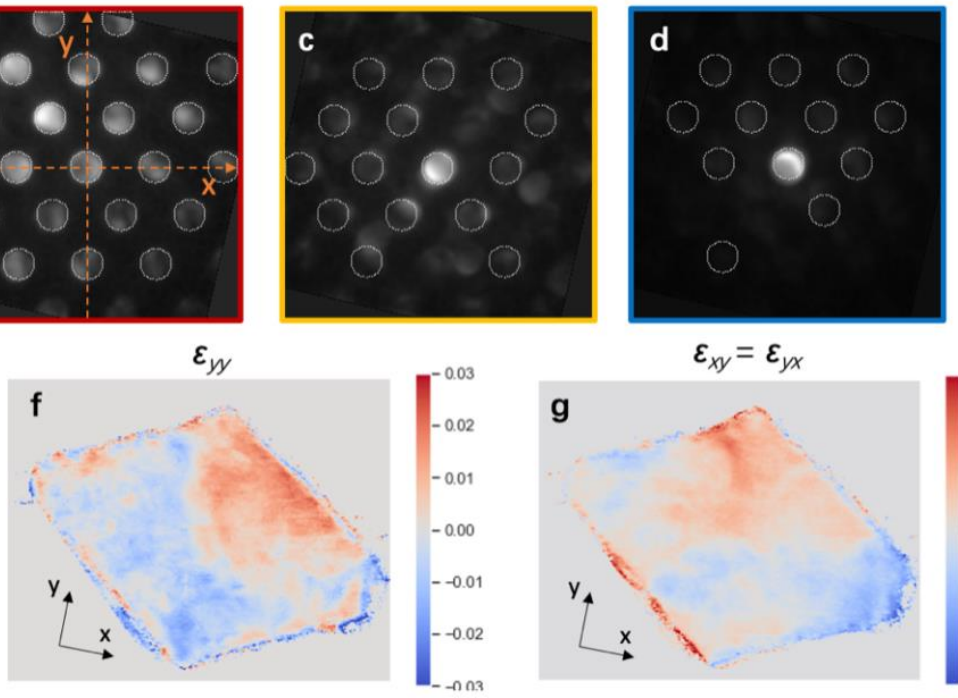

$$
\varepsilon_{x y}=\varepsilon_{y x}
$$

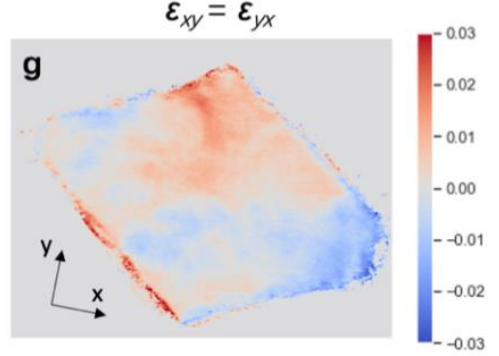

Figure 2. (a) The bright field STEM image of a Pd@Pt octahedral nanoparticle. (b) (d) Disk detection and lattice fitting results at corresponding points in (a), with $\mathrm{x}$ along the (002) direction and y along the (220) direction. (e-g) Normal and shear strain mappings of the nanoparticle.

\section{References}

[1] C. Ophus, Four-Dimensional Scanning Transmission Electron Microscopy (4D-STEM): From Scanning Nanodiffraction to Ptychography and Beyond, Microsc Microanal. 25 (2019) 563-582. https://doi.org/10.1017/S1431927619000497.

[2] I. Lobato, D. Van Dyck, MULTEM: A new multislice program to perform accurate and fast electron diffraction and imaging simulations using Graphics Processing Units with CUDA, Ultramicroscopy. 156 (2015) 9-17. https://doi.org/10.1016/j.ultramic.2015.04.016.

[3] K. Müller, A. Rosenauer, M. Schowalter, J. Zweck, R. Fritz, K. Volz, Strain Measurement in Semiconductor Heterostructures by Scanning Transmission Electron Microscopy, Microsc Microanal. 18 (2012) 995-1009. https://doi.org/10.1017/S1431927612001274.

[4] J.L. Rouvière, E. Sarigiannidou, Theoretical discussions on the geometrical phase analysis, Ultramicroscopy. 106 (2005) 1-17. https://doi.org/10.1016/j.ultramic.2005.06.001.

[5] This work was supported by start-up fund from the College of Engineering and the Department of Materials Science and Engineering at North Carolina State University. Electron microscopy was performed at the Analytical Instrumentation Facility (AIF) at North Carolina State University, which is supported by the State of North Carolina and the National Science Foundation (award number ECCS-2025064). The AIF is a member of the North Carolina Research Triangle Nanotechnology Network (RTNN), a site in the National Nanotechnology Coordinated Infrastructure (NNCI). 